\title{
A Preliminary Study of microRNA-208b after Acute Myocardial Infarction: Impact on 6-Month Survival
}

\author{
Mostafa Alavi-Moghaddam,,2 Mohammad Chehrazi, ${ }^{3}$ Shamila D. Alipoor, \\ Maryam Mohammadi, ${ }^{1}$ Alireza Baratloo, ${ }^{5}$ Mohammad Parsa Mahjoub, ${ }^{6}$ \\ Mehrnaz Movasaghi, ${ }^{7}$ Johan Garssen, ${ }^{8,9}$ Ian M. Adcock $\left.\mathbb{D}\right)^{10,11}$ and Esmaeil Mortaz $\mathbb{D}^{7,12}$ \\ ${ }^{1}$ Emergency Medicine Department, Imam Hossein Hospital, Shahid Beheshti University of Medical Sciences, Tehran, Iran \\ ${ }^{2}$ Clinical Immunology Department, National Research Institute of Tuberculosis and Lung Diseases (NRITLD), Shahid Beheshti \\ University of Medical Sciences, Tehran, Iran \\ ${ }^{3}$ Department of Epidemiology and Reproductive Health, Reproductive Epidemiology Research Center, Royan Institute for \\ Reproductive Biomedicine, ACECR, Tehran, Iran \\ ${ }^{4}$ Institute of Medical Biotechnology, Molecular Medicine Department, National Institute of Genetic Engineering and Biotechnology \\ (NIGEB), Tehran, Iran \\ ${ }^{5}$ Emergency Medicine Department, Tehran University of Medical Sciences, Tehran, Iran \\ ${ }^{6}$ Department of Cardiology, Imam Hossein Hospital, Shahid Beheshti University of Medical Sciences, Tehran, Iran \\ ${ }^{7}$ Clinical Tuberculosis and Epidemiology Research Center, National Research Institute for Tuberculosis and Lung Disease (NRITLD), \\ Shahid Beheshti University of Medical Sciences, Tehran, Iran \\ ${ }^{8}$ Division of Pharmacology, Faculty of Science, Utrecht Institute for Pharmaceutical Sciences, Utrecht University, Utrecht, Netherlands \\ ${ }^{9}$ Nutricia Research Centre for Specialized Nutrition, Utrecht, Netherlands \\ ${ }^{10}$ Cell and Molecular Biology Group, Airways Disease Section, Faculty of Medicine, National Heart and Lung Institute, Imperial \\ College London, London, UK \\ ${ }^{11}$ Priority Research Centre for Asthma and Respiratory Disease, Hunter Medical Research Institute, University of Newcastle, \\ Newcastle, NSW, Australia \\ ${ }^{12}$ Department of Immunology, School of Medicine, Shahid Beheshti University of Medical Sciences, Tehran, Iran
}

Correspondence should be addressed to Esmaeil Mortaz; e.mortaz@uu.nl

Received 30 January 2018; Accepted 23 April 2018; Published 27 May 2018

Academic Editor: Andrea Salzano

Copyright @ 2018 Mostafa Alavi-Moghaddam et al. This is an open access article distributed under the Creative Commons Attribution License, which permits unrestricted use, distribution, and reproduction in any medium, provided the original work is properly cited.

Introduction. miRNAs contribute to a variety of essential biological processes including development, proliferation, differentiation, and apoptosis. Circulating microRNAs are very stable and have shown potential as biomarkers of cardiovascular disease. microRNA-208b expression was increased in the blood of patients with acute myocardial infarction (AMI) and has been proposed as a biomarker for early diagnosis. In this pilot study, we investigate the potential of circulating miR-208b as a prognostic biomarker of 6-month survival in AMI patients. Methods. Plasma samples from 21 patients and 8 age- and gendermatched healthy adults were collected, and circulating levels of miR-208b were detected using quantitative real-time PCR. Results. miR-208b levels were higher in healthy control subjects (9.6-fold; $P \leq 0.05$ ). Within the AMI patients, the levels of miR$208 \mathrm{~b}$ were significantly lower in the survivor versus nonsurvivor group (fold change $=6.51$ and 14.1, resp.; $P \leq 0.05$ ). The Kaplan-Meier curve revealed that the 6-month survival time was significantly higher among AMI patients with a relative expression of miR-208b lower than 12.38. The hazard ratio (HR) for the relative expression of miR-208b $(<12.38$ was the reference) was 5.08 (95\% CI: $1.13-22.82 ; P=0.03)$. Conclusion. Our results showed that elevated miR-208b expression was associated with reduced long-term survival in AMI patients. These pilot data indicate the need for a large follow-up study to confirm whether miR-208b can be used as a predictor of 6-month survival time after AMI. 


\section{Introduction}

Acute myocardial infarction (AMI) occurs as a result of the acute necrosis of myocardial tissue following persistent and severe ischemia [1]. AMI is one of the most common cardiovascular diseases and one of the leading causes of mortality and morbidity across the globe. 17 million people die annually of cardiovascular diseases with 10 million being in developing countries [2-4]. Patients with a comorbid diagnosis of AMI had two to three times the case-fatality rate of patients in whom AMI was a primary diagnosis [5]. It is predicted that cardiovascular diseases will constitute $36 \%$ of all deaths globally in 2020 [6]. Some conventional biomarkers, such as blood troponins, cardiac myoglobin, and creatine kinase$\mathrm{MB}(\mathrm{CK}-\mathrm{MB})$ are currently used for clinical diagnosis of AMI [7].

microRNAs (miRNAs) are small (19-25 nucleotides in length), noncoding, and highly conserved RNA molecules which are involved in the regulation of gene expression. The regulatory functions of miRNAs are achieved through the RNA-induced silencing complex [8]. miRNAs control a variety of essential biological processes including development, proliferation, differentiation, and apoptosis [9]. Dysregulated tissue expression of miRNAs contributes to various diseases such as cancer and cardiovascular disease [10-12]. Recent studies demonstrated that miRNAs play a crucial role in AMI mechanisms such as atherosclerotic plaque rupture, blood platelet aggregation, and necrosis of heart cells after blockage of the coronary artery [13].

Many miRNAs are remarkably stable and easily detectable in the peripheral blood or plasma $[14,15]$. The levels of circulating miRNAs may differ under pathological conditions [16-18]. This suggests plasma miRNA concentrations may be used as superior biomarkers for the diagnosis and prognosis of diseases in humans $[19,20]$. The levels of several miRNAs such as miR-1, miR-133a, miR-208b, miR-499, and miR-328 are altered in the blood and plasma during AMI [21-26]. miR-149, miR-499, and miR-208b are increased immediately after percutaneous coronary intervention (PCI) and therefore have promise as diagnostic and prognostic biomarkers in AMI [27]. miR-208 is produced exclusively in the rat myocardium and is considered as a biomarker of myocardial injury in rats [28]. The same study reported that the plasma level of miR-499 may also be a useful biomarker of myocardial infarction in humans [28]. The present study aimed to investigate the potential prognostic value of circulating miR-208b in AMI patients with respect to 6-month survival time.

\section{Methods}

2.1. Patient Characteristics. This pilot prospective prognostic study recruited AMI patients sequentially referred to the Imam Hossein Hospital affiliated to the Shahid Beheshti University of Medical Sciences between January and December 2016. The study was approved by the Research Ethics Committee of Shahid Beheshti University of Medical Sciences at Tehran, Iran, and all patients gave informed consent.
Patients with acute ischemic chest pain, abnormal electrocardiogram (pathological Q wave and ST-segment elevation), and increased levels of troponin and creatine kinase greater than 2 times the upper limit of the normal range with a diagnosis of AMI were enrolled into the study. Patients with a previous history of venous thrombolytic injection or receiving anticoagulant, previous $\mathrm{MI}$ or PCI, hematological diseases, acute or chronic infection, significant hepatic dysfunction, renal failure, or known or cured malignancy were excluded. The patients were admitted to hospital no more than $12 \mathrm{~h}$ after the emergence of symptoms, and blood samples were collected immediately after admission. A cutoff value of $55 \%$ was used for the ejection fraction (EF). If the diagnosis of AMI was confirmed, then the blood samples were submitted to the reference laboratory for miRNA analysis.

Five-milliliter venous blood samples of patients with AMI were collected in EDTA anticoagulant tubes at admission. Samples were centrifuged at $3000 \times \mathrm{g}$ for $10 \mathrm{~min}$ at $4^{\circ} \mathrm{C}$, and then the supernatant was isolated and centrifuged at $12,000 \times \mathrm{g}$ for $10 \mathrm{~min}$ at $4^{\circ} \mathrm{C}$. Plasma was collected and stored at $-80^{\circ} \mathrm{C}$ until RNA extraction. Moreover, 8 age- and gendermatched healthy volunteers with normal electrocardiograms and no history of cardiovascular diseases were recruited as a control group.

2.2. RNA Extraction and cDNA Synthesis. Serum-free miRNAs in patients and the control group were extracted using an RNA extraction kit (Exiqon, Vedbæk, Denmark). Extracted RNA was reverse transcribed using the miRCURY LNA Universal RT microRNA cDNA Synthesis Kit (Exiqon) according to the manufacturer's instructions.

\subsection{Real-Time Quantitative PCR Analysis}

2.3.1. Quantitative Reverse Transcription-Polymerase Chain Reaction ( $q R T-P C R)$. Real-time PCR assays were performed using the ExiLENT SYBR ${ }^{\circledR}$ Green Master Mix Kit (Exiqon). LNA primers were purchased from Exiqon. cDNA was diluted $10 \mathrm{x}$ and added to the PCR reactions according to the manufacturer's instructions. The real-time PCR program included the following steps: an initial denaturation step at $95^{\circ} \mathrm{C}$ for $10 \mathrm{~min}$ and 50 cycles of amplification that consisted of a denaturation step $\left(10 \mathrm{~s}\right.$ at $\left.95^{\circ} \mathrm{C}\right)$ and an annealing step $\left(60 \mathrm{~s}\right.$ at $\left.60^{\circ} \mathrm{C}\right)$. The expression levels of miR-208b were normalized to the level of miR-16 as control using the efficiency-corrected calculation models of the Pfaffle method [29].

$$
\text { Ratio }=\frac{(\text { Etarget }) \Delta \text { Ct target }(\text { control }- \text { sample })}{(\text { ERef }) \Delta \text { Ct Ref }(\text { control }- \text { sample })} .
$$

2.4. Statistical Analysis. Data were presented using mean (SD) and frequency and 95\% confidence interval (95\% CI). Independent sample $t$-tests or Mann-Whitney $U$ tests and chi-square test were used to investigate the differences in continuous and categorical variables, respectively. The Kaplan-Meier method was used for depicting univariate survival curves illustrating the association between the biomarker expression and disease-specific survival (DSS). DSS 
TABLE 1: Clinical features and risk factors of the cohort.

\begin{tabular}{|c|c|c|c|c|}
\hline Characteristics & All $(n=21)$ & Survivor $(n=14)$ & Nonsurvivor $(n=7)$ & $P$ value \\
\hline Age & $62.71(12.75)$ & $58.57(11.37)$ & $71(11.88)$ & 0.15 \\
\hline $\operatorname{Sex}(M / F)$ & $15 / 6$ & $9 / 5$ & $6 / 1$ & 0.31 \\
\hline Smoking (yes/no) & $8 / 13$ & $5 / 9$ & $3 / 4$ & 0.75 \\
\hline Diabetes (yes/no) & $4 / 17$ & $3 / 11$ & $1 / 6$ & 0.69 \\
\hline Hypertension (yes/no) & $13 / 8$ & $8 / 6$ & $5 / 2$ & 0.52 \\
\hline Hyperlipidemia (yes/no) & $10 / 11$ & $6 / 8$ & $4 / 3$ & 0.54 \\
\hline Cardiac troponin $\mathrm{T}(\mathrm{ng} / \mathrm{mL})$ & $10.08(10.18)$ & $10.54(10.20)$ & $9.15(10.89)$ & 0.77 \\
\hline Decrease in $\mathrm{EF}^{*}$ (yes/no) & $6 / 13$ & $4 / 10$ & $2 / 3$ & 0.64 \\
\hline Relative expression & $8.63(12.83)$ & $4.58(4.85)$ & $16.72(19.58)$ & 0.04 \\
\hline
\end{tabular}

${ }^{*}$ Cutoff $=0.55$.

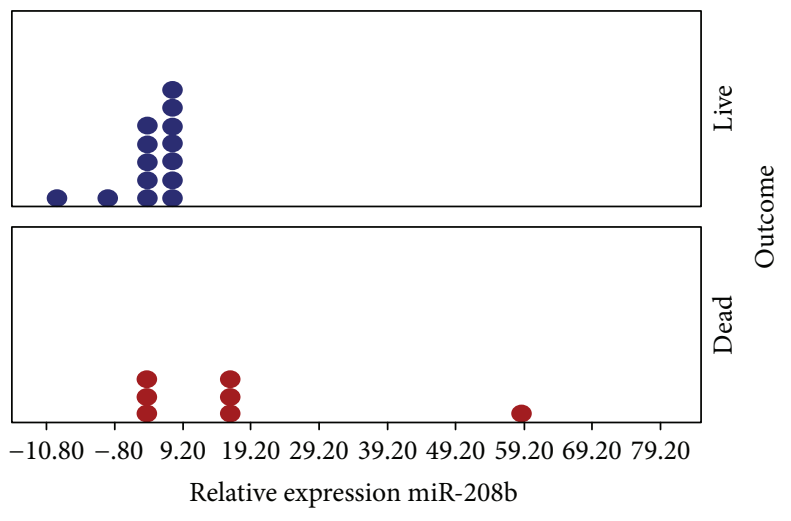

(a)

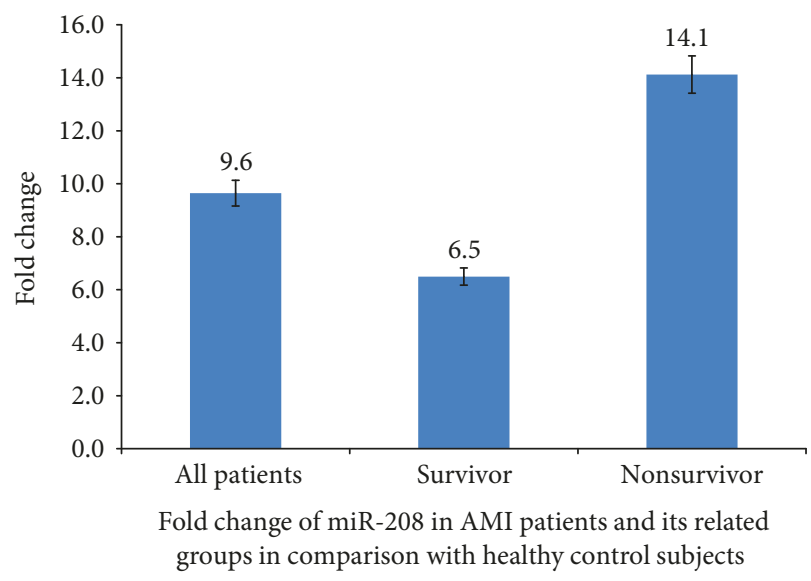

(b)

FIGURE 1: (a) Relative expression of circulating miR-208b in AMI patients in the survivor and nonsurvivor groups $(P=0.03)$. The expression of miR-208b was 9.6-fold higher in the AMI group compared with the healthy control subjects. (b) The relative expression of miR-208b was increased in both survivor and nonsurvivor groups in comparison to healthy subjects (fold change $=6.51$ and 14.1, resp.). Plasma samples were collected upon admission no more than $24 \mathrm{~h}$ after AMI onset (in all cases, $P$ value $\leq 0.05$ ).

was defined from the date of enrollment until the time of AMI death. Statistical significance between the survival curves was assessed utilizing the log-rank test. The Cox proportional hazard model was used to estimate the hazard ratio of death for miR-208b. The cutoff value was determined based on the Youden index. $P$ values less than 0.05 were considered statistically significant for all analyses. All statistical analyses were performed using the statistical package IBM SPSS, version 21 (SPSS Inc., Chicago, IL, USA).

\section{Results}

3.1. Clinical Characteristics of Patients. Among the 21 patients diagnosed with AMI, 7 patients died within six months of diagnosis. Both the nonsurvivor and the survivor groups were predominantly male (6/7 and $9 / 14$, resp.). The demographics of the patients in this study are shown in Table 1. No significant differences were observed in the personal history including hypertension, hyperlipidemia, diabetes, cardiac troponin $\mathrm{T}$, and the left ventricular ejection fraction (EF) and smoking between nonsurvivor and survivor patients.
3.2. Assessment of the Circulating miR-208b Levels. The level of miR-208b was measured in the plasma of 21 AMI patients according to the survival time as well as in the healthy controls. The expression of miR-208b was significantly greater in the AMI group compared with healthy control subjects (fold change $=9.6, P \leq 0.05$ ) (Figure 1). As shown in Figure 1, the relative expression of $\mathrm{miR}-208 \mathrm{~b}$ was increased in both survivor and nonsurvivor groups as compared to healthy subjects (fold change $=6.51$ and 14.1 , resp.; $P \leq 0.05$ ). There was no effect of age on the miR-208b level (Spearman $r=0.2049$ and $P$ value (two-tailed) $=0.4148$ ).

The result also showed a significant difference in the plasma level of miR-208b between the survivor and nonsurvivor groups in AMI patients (Figure 1). The plasma level of circulating miR-208b in nonsurvivors was 2.1-fold higher than that in survivors (Figure 1).

3.3. Survival Analysis. The influence of the clinical characteristics on the median and 6-month survival is presented in Table 2. Only the relative expression of miR-208b $(P=0.02)$ was a significant prognosticator. Table 3 presents the results from the Cox regression analyses regarding the 
TABLE 2: Clinical variables as predictors of the survival all AMI patients and differentiated into lower and upper 12.38 subgroups $(N=21,17$, and 4 , resp., univariate analyses; log-rank test) in 21 AMI patients.

\begin{tabular}{|c|c|c|c|c|}
\hline Characteristics & $\begin{array}{l}\text { Patient } N \\
(\mathrm{NOE})\end{array}$ & $\begin{array}{c}\text { Median } \\
\text { survival } \\
\text { (days) }\end{array}$ & $\begin{array}{c}\text { 6-month } \\
\text { survival (\%) }\end{array}$ & $P$ value \\
\hline \multicolumn{5}{|l|}{$\begin{array}{l}\text { Relative } \\
\text { expression }\end{array}$} \\
\hline$<12.38$ & $17(3)$ & NR & 66.2 & \multirow{2}{*}{0.02} \\
\hline$\geq 12.38$ & $4(4)$ & 43 & 0 & \\
\hline \multicolumn{5}{|l|}{ Gender } \\
\hline Female & $6(1)$ & 182 & 75 & \multirow{2}{*}{0.32} \\
\hline Male & $15(6)$ & NR & 36.7 & \\
\hline \multicolumn{5}{|l|}{ Smoking } \\
\hline Yes & $8(3)$ & 182 & 47 & \multirow{2}{*}{0.81} \\
\hline No & $13(4)$ & 157 & 37.5 & \\
\hline \multicolumn{5}{|l|}{ Diabetes } \\
\hline Yes & $4(1)$ & NR & 75 & \multirow{2}{*}{0.69} \\
\hline No & $17(6)$ & 182 & 34.3 & \\
\hline \multicolumn{5}{|l|}{ Hypertension } \\
\hline Yes & $13(5)$ & 182 & 42.7 & \multirow{2}{*}{0.75} \\
\hline No & $8(2)$ & 157 & 43.8 & \\
\hline \multicolumn{5}{|l|}{ Hyperlipidemia } \\
\hline Yes & $10(4)$ & NR & 52.5 & \multirow{2}{*}{0.32} \\
\hline No & $11(3)$ & 182 & 45.5 & \\
\hline \multicolumn{5}{|l|}{ Decrease in $E F^{*}$} \\
\hline Yes & $6(2)$ & 182 & 41.7 & \multirow{2}{*}{0.85} \\
\hline No & $13(3)$ & NR & 51.3 & \\
\hline
\end{tabular}

NOE: number of events; NR: not reached. ${ }^{*}$ Cutoff $=0.55$.

clinical variables and their impact on the survival. All the variables showed no significant relation with survival in univariate analyses except the relative expression of miR$208 \mathrm{~b}$. To compare survival function according to levels of miR-208b, we put a cutoff point of 12.38 which was obtained by using the Youden index. High relative expression of miR-208b was the most significant negative prognostic factor in our patient cohort (HR: 5.08; 95\% CI: $1.13-22.82 ; P=0.03$ ) (Figure 2).

\section{Discussion}

In this study, we investigated the prognostic value of miR$208 \mathrm{~b}$ to predict the 6-month survival time for patients with ST-elevation myocardial infarction (STEMI). qRT-PCR analysis confirmed that baseline plasma levels of miR-208b were greater in AMI patients compared with healthy controls. An important finding in this study was that the levels of miR-208b on admission had a significant ability to predict 6-month survival time. The survival curves indicated that a relative expression cutoff of 12.38 for circulating miR-208b clearly distinguished the survival odds.
TABLE 3: Results of Cox regression analyses for clinical variables and miR-208 relative expression among AMI patients.

\begin{tabular}{|c|c|c|c|}
\hline Characteristics & HR & $95 \% \mathrm{CI}$ & $P$ value \\
\hline Age & 1.04 & $(0.98-1.10)$ & 0.15 \\
\hline Cardiac troponin $T(n g / m L)$ & 0.97 & $(0.87-1.05)$ & 0.33 \\
\hline \multicolumn{4}{|l|}{ Relative expression } \\
\hline$<12.38$ & Reference & & \multirow{2}{*}{0.03} \\
\hline$\geq 12.38$ & 5.08 & $(1.13-22.82)$ & \\
\hline \multicolumn{4}{|l|}{ Gender } \\
\hline Female & Reference & & \multirow{2}{*}{0.35} \\
\hline Male & 2.81 & $(0.33-23.91)$ & \\
\hline \multicolumn{4}{|l|}{ Smoking } \\
\hline No & Reference & & \multirow{2}{*}{0.81} \\
\hline Yes & 1.21 & $(0.27-5.48)$ & \\
\hline \multicolumn{4}{|l|}{ Diabetes } \\
\hline No & Reference & & \multirow{2}{*}{0.69} \\
\hline Yes & 0.66 & $(0.08-5.48)$ & \\
\hline \multicolumn{4}{|l|}{ Hypertension } \\
\hline No & Reference & & \multirow{2}{*}{0.75} \\
\hline Yes & 1.30 & $(0.25-6.77)$ & \\
\hline \multicolumn{4}{|l|}{ Hyperlipidemia } \\
\hline No & Reference & & \multirow{2}{*}{0.33} \\
\hline Yes & 2.16 & $(0.46-10.13)$ & \\
\hline \multicolumn{4}{|l|}{ Decrease in $E F^{*}$} \\
\hline No & Reference & & \multirow{2}{*}{0.85} \\
\hline Yes & 1.19 & $(0.19-7.53)$ & \\
\hline
\end{tabular}

HR: hazard ratio. ${ }^{*}$ Cutoff $=0.55$.

The diagnosis and treatment of AMI patients is of paramount importance, and predictive factors could have widespread application in clinical practice [2]. Cardiac troponins and creatine kinase-MB are the most common biomarkers for AMI diagnosis. However, their detection may be limited in some cases. Thus, measuring the levels of circulating miRNAs might provide an additional specific biomarker for the diagnosis and treatment of AMI. In addition, higher plasma levels of miR-208b have also been significantly associated with the risk of death during a 6-month follow-up in acute coronary syndrome (ACS) [30]. Thus, a combination of plasma miR-208b detection with clinical characterization may give greater prognosis of 6-month survival in patients with different types of heart disease.

miR-208b is encoded by intron 31 within the MYH7 gene and regulates the expression of its host gene via the Sox6 transcription factor. The MYH7 gene encodes the beta- $(\beta-)$ myosin heavy chain 7 that is found in the heart (cardiac) muscle [31]. MiR-208b is considered a cardiac-specific miRNA with an important role in human heart function and cardiopathology [32]. During the early stages of AMI, this miRNA might leak out of the necrotic myocardium and be released into the circulation [33]. Cardiomyocyteenriched miRNAs have been recently considered as the potential diagnostic biomarkers in AMI due to their rapid release, cardiac selectivity, and plasma stability [30]. Previous 


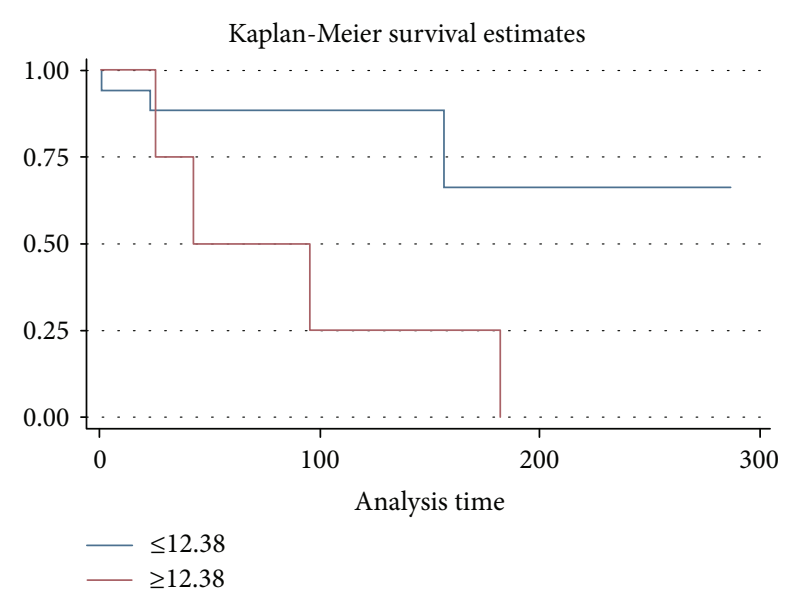

FIGURE 2: Kaplan-Meier curve displaying the survival in relation to high or low miR-208b relative expression.

studies reported that the plasma level of some miRNAs such as miR-1, miR-133a, miR-133b, miR-208a, and miR-499 was significantly elevated in patients with STEMI compared to healthy controls and patients with chest pain but normal coronary angiograms [32-34].

Plasma levels of miR-208b have been previously studied in relation to their predictive value in AMI [35]. Plasma miR-208b levels were higher in AMI patients, and ROC analysis gave AUC values of 0.72. An even greater predictive ROC value (0.94) for miR-208b in AMI was reported by Corsten and colleagues [33]. Several other studies have reported elevated miR-208b levels in AMI compared with healthy control subjects which have implied a role for miR-208b as a diagnostic marker [36-38]. However, none of these studies considered the rate of survival post-AMI.

A rapid increase in the level of circulating miR-208b after myocardial infarction is correlated with decreased systolic function, increased rejection fraction, and increased expression of markers of cardiomyocyte necrosis [39]. It is plausible that following myocardial damage, cardio-enriched miRNAs such as miR-208b are released into the bloodstream from necrotic cardiomyocytes which subsequently have a paracrine effect on the heart [40]. For example, miR-208b may exacerbate the deleterious conditions within the myocardium post-MI and increase the risk of death or development of heart failure [41]. As such, strategies designed to inhibit miR-208b may be of therapeutic value. In support of this concept, inhibition of miR-208a improves cardiac function and survival during heart failure [31] in addition to acting as a potential noninvasive biomarker of myocardial injury [31].

The effect of age and age-related diseases such as cancer and cardiovascular disease on the expression of circulating miRNAs has been previously examined [42]. An agedependent decrease in miRNA expression in peripheral blood mononuclear cells (PBMCs) was seen along with lower serum levels of miR-151a-5p, miR-181a-5p, and miR-1248 [42-44]. Our data failed to show a clear effect of age on miR-208b expression, but further studies using subjects with a greater age range should be investigated.

\section{Conclusion}

Our result confirms previous studies demonstrating a possible role of miR-208b as a candidate biomarker for AMI diagnosis [27] and extends this to show that the precise level of miR-208b in these patients on admission was a good indicator of 6-month survival. Thus, the relative expression of miR208b was significantly increased in AMI patients who died within 6 months compared to those AMI patients who survived, but larger studies are required to confirm this.

5.1. Limitations. There were some limitations to our study. Our findings are based on a small sample size, and further research with a larger sample size with a longer follow-up time is required to obtain accurate and reproducible results. Repeated measurements of miR-208b levels at more time points would also enhance the reliability of our findings.

\section{Data Availability}

The data used to support the findings of this study are available from the corresponding author upon request.

\section{Conflicts of Interest}

There is no conflict of interest among the authors.

\section{Acknowledgments}

The authors greatly appreciate the support of the Clinical Research Development Unit, Imam Hossein Hospital, Shahid Beheshti University of Medical Sciences, Tehran, Iran, in the submission and revision of the final copy of the manuscript. IMA is supported by Wellcome Trust grant 093080/Z/10/Z.

\section{References}

[1] H. D. White and D. P. Chew, "Acute myocardial infarction," The Lancet, vol. 372, no. 9638, pp. 570-584, 2008.

[2] S. Yusuf, S. Reddy, S. Ounpuu, and S. Anand, "Global burden of cardiovascular diseases: part II: variations in cardiovascular disease by specific ethnic groups and geographic regions and prevention strategies," Circulation, vol. 104, no. 23, pp. 2855-2864, 2001.

[3] N. R. Sodha, R. T. Clements, J. Feng et al., "Hydrogen sulfide therapy attenuates the inflammatory response in a porcine model of myocardial ischemia/reperfusion injury," The Journal of Thoracic and Cardiovascular Surgery, vol. 138, no. 4, pp. 977-984, 2009.

[4] S. H. Jee, M. Kivimaki, H. C. Kang, I. S. Park, J. M. Samet, and G. D. Batty, "Cardiovascular disease risk factors in relation to suicide mortality in Asia: prospective cohort study of over one million Korean men and women," European Heart Journal, vol. 32, no. 22, pp. 2773-2780, 2011.

[5] P. Asaria, P. Elliott, M. Douglass et al., "Acute myocardial infarction hospital admissions and deaths in England: a national follow-back and follow-forward record-linkage study," The Lancet Public Health, vol. 2, no. 4, pp. e191e201, 2017. 
[6] R. Zhang, C. Lan, H. Pei, G. Duan, L. Huang, and L. Li, "Expression of circulating miR-486 and miR-150 in patients with acute myocardial infarction," BMC Cardiovascular Disorders, vol. 15, no. 1, p. 51, 2015.

[7] S. J. Aldous, "Cardiac biomarkers in acute myocardial infarction," International Journal of Cardiology, vol. 164, no. 3, pp. 282-294, 2013.

[8] S. D. Alipoor, I. M. Adcock, J. Garssen et al., "The roles of miRNAs as potential biomarkers in lung diseases," European Journal of Pharmacology, vol. 791, pp. 395-404, 2016.

[9] S. D. Alipoor, E. Mortaz, J. Garssen, M. Movassaghi, M. Mirsaeidi, and I. M. Adcock, "Exosomes and exosomal miRNA in respiratory diseases," Mediators of Inflammation, vol. 2016, Article ID 5628404, 11 pages, 2016.

[10] E. M. Small and E. N. Olson, "Pervasive roles of microRNAs in cardiovascular biology," Nature, vol. 469, no. 7330, pp. 336342, 2011

[11] P. S. Meltzer, "Cancer genomics: small RNAs with big impacts," Nature, vol. 435, no. 7043, pp. 745-746, 2005.

[12] D. Sayed and M. Abdellatif, "MicroRNAs in development and disease," Physiological Reviews, vol. 91, no. 3, pp. 827-887, 2011.

[13] T. Sun, Y. H. Dong, W. du et al., "The role of microRNAs in myocardial infarction: from molecular mechanism to clinical application," International Journal of Molecular Sciences, vol. 18, no. 4, p. 745, 2017.

[14] E. van Rooij, N. Liu, and E. N. Olson, "MicroRNAs flex their muscles," Trends in Genetics, vol. 24, no. 4, pp. 159-166, 2008.

[15] M. Lagos-Quintana, R. Rauhut, A. Yalcin, J. Meyer, W. Lendeckel, and T. Tuschl, "Identification of tissue-specific microRNAs from mouse," Current Biology, vol. 12, no. 9, pp. 735-9, 2002.

[16] V. P. van Empel, L. J. De Windt, and P. A. da Costa Martins, "Circulating miRNAs: reflecting or affecting cardiovascular disease?," Current Hypertension Reports, vol. 14, no. 6, pp. 498-509, 2012.

[17] W. P. Kloosterman and R. H. Plasterk, "The diverse functions of microRNAs in animal development and disease," Developmental Cell, vol. 11, no. 4, pp. 441-450, 2006.

[18] P. S. Mitchell, R. K. Parkin, E. M. Kroh et al., "Circulating microRNAs as stable blood-based markers for cancer detection," Proceedings of the National Academy of Sciences of the United States of America, vol. 105, no. 30, pp. 10513-10518, 2008.

[19] S. Gilad, E. Meiri, Y. Yogev et al., "Serum microRNAs are promising novel biomarkers," PLoS One, vol. 3, no. 9, article e3148, 2008.

[20] X. Chen, Y. Ba, L. Ma et al., "Characterization of microRNAs in serum: a novel class of biomarkers for diagnosis of cancer and other diseases," Cell Research, vol. 18, no. 10, pp. 9971006, 2008.

[21] M. Häntzsch, A. Tolios, F. Beutner et al., "Comparison of whole blood RNA preservation tubes and novel generation RNA extraction kits for analysis of mRNA and miRNA profiles," PLoS One, vol. 9, no. 12, article e113298, 2014.

[22] H. Zhou, X. Y. He, S. W. Zhuang et al., "Clinical and procedural predictors of no-reflow in patients with acute myocardial infarction after primary percutaneous coronary intervention," World Journal of Emergency Medicine, vol. 5, no. 2, pp. 96-102, 2014.

[23] G. Long, F. Wang, Q. Duan et al., "Human circulating microRNA-1 and microRNA-126 as potential novel indicators for acute myocardial infarction," International Journal of Biological Sciences, vol. 8, no. 6, pp. 811-818, 2012.

[24] E. Boštjančič, N. Zidar, D. Štajer, and D. Glavač, "MicroRNAs $m i R-1, m i R-133 a, m i R-133 b$ and $m i R-208$ are dysregulated in human myocardial infarction," Cardiology, vol. 115, no. 3, pp. 163-169, 2010.

[25] J. Xiao, B. Shen, J. Li et al., "Serum microRNA-499 and microRNA-208a as biomarkers of acute myocardial infarction," International Journal of Clinical and Experimental Medicine, vol. 7, no. 1, pp. 136-141, 2014.

[26] F. He, P. Lv, X. Zhao et al., "Predictive value of circulating miR-328 and miR-134 for acute myocardial infarction," Molecular and Cellular Biochemistry, vol. 394, no. 1-2, pp. 137-144, 2014.

[27] A. S. Sayed, K. Xia, T.-L. Yang, and J. Peng, "Circulating microRNAs: a potential role in diagnosis and prognosis of acute myocardial infarction," Disease Markers, vol. 35, no. 5, 566 pages, 2013.

[28] T. Adachi, M. Nakanishi, Y. Otsuka et al., "Plasma microRNA 499 as a biomarker of acute myocardial infarction," Clinical Chemistry, vol. 56, no. 7, pp. 1183-1185, 2010.

[29] M. W. Pfaffl, "A new mathematical model for relative quantification in real-time RT-PCR," Nucleic Acids Research, vol. 29, no. 9, article e45, 2001.

[30] C. Widera, S. K. Gupta, J. M. Lorenzen et al., "Diagnostic and prognostic impact of six circulating microRNAs in acute coronary syndrome," Journal of Molecular and Cellular Cardiology, vol. 51 , no. 5 , pp. $872-5,2011$.

[31] R. L. Montgomery, T. G. Hullinger, H. M. Semus et al., "Therapeutic inhibition of miR-208a improves cardiac function and survival during heart failure," Circulation, vol. 124, no. 14, pp. 1537-1547, 2011.

[32] G. K. Wang, J. Q. Zhu, J. T. Zhang et al., "Circulating microRNA: a novel potential biomarker for early diagnosis of acute myocardial infarction in humans," European Heart Journal, vol. 31, no. 6, pp. 659-666, 2010.

[33] M. F. Corsten, R. Dennert, S. Jochems et al., "Circulating MicroRNA-208b and microRNA-499 reflect myocardial damage in cardiovascular disease," Circulation. Cardiovascular Genetics, vol. 3, no. 6, pp. 499-506, 2010.

[34] Y. D'Alessandra, P. Devanna, F. Limana et al., "Circulating microRNAs are new and sensitive biomarkers of myocardial infarction," European Heart Journal, vol. 31, no. 22, pp. 2765-2773, 2010.

[35] X. Liu, Z. Fan, T. Zhao et al., "Plasma miR-1, miR-208, miR499 as potential predictive biomarkers for acute myocardial infarction: an independent study of Han population," Experimental Gerontology, vol. 72, pp. 230-238, 2015.

[36] Y. Goren, E. Meiri, C. Hogan et al., "Relation of reduced expression of miR-150 in platelets to atrial fibrillation in patients with chronic systolic heart failure," The American Journal of Cardiology, vol. 113, no. 6, pp. 976-981, 2014.

[37] K. Schlosser, R. J. White, and D. J. Stewart, "miR-26a linked to pulmonary hypertension by global assessment of circulating extracellular microRNAs," American Journal of Respiratory and Critical Care Medicine, vol. 188, no. 12, pp. 1472-1475, 2013.

[38] C. Li, X. Chen, J. Huang, Q. Sun, and L. Wang, "Clinical impact of circulating miR-26a, miR-191, and miR-208b in plasma of patients with acute myocardial infarction," European Journal of Medical Research, vol. 20, no. 1, p. 58, 2015. 
[39] O. Gidlöf, P. Andersson, J. van der Pals, M. Götberg, and D. Erlinge, "Cardiospecific microRNA plasma levels correlate with troponin and cardiac function in patients with ST elevation myocardial infarction, are selectively dependent on renal elimination, and can be detected in urine samples," Cardiology, vol. 118, no. 4, pp. 217-226, 2011.

[40] A. Zernecke, K. Bidzhekov, H. Noels et al., "Delivery of microRNA-126 by apoptotic bodies induces CXCL12dependent vascular protection," Science Signaling, vol. 2, no. 100 , article ra81, 2009.

[41] O. Gidlof, O. Gidlöf, J. Gustav Smith et al., "Circulating cardio-enriched microRNAs are associated with long-term prognosis following myocardial infarction," BMC Cardiovascular Disorders, vol. 13, no. 1, p. 12, 2013.

[42] N. N. Hooten, M. Fitzpatrick, W. H. Wood et al., "Age-related changes in microRNA levels in serum," Aging, vol. 5, no. 10, pp. 725-740, 2013.

[43] N. N. Hooten, K. Abdelmohsen, M. Gorospe, N. Ejiogu, A. B. Zonderman, and M. K. Evans, "microRNA expression patterns reveal differential expression of target genes with age," PLoS One, vol. 5, no. 5, article e10724, 2010.

[44] S. Hatse, B. Brouwers, B. Dalmasso et al., "Circulating microRNAs as easy-to-measure aging biomarkers in older breast cancer patients: correlation with chronological age but not with fitness/frailty status," PLoS One, vol. 9, no. 10, article e110644, 2014. 


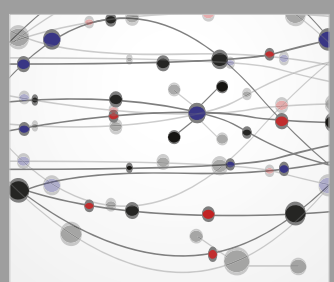

The Scientific World Journal
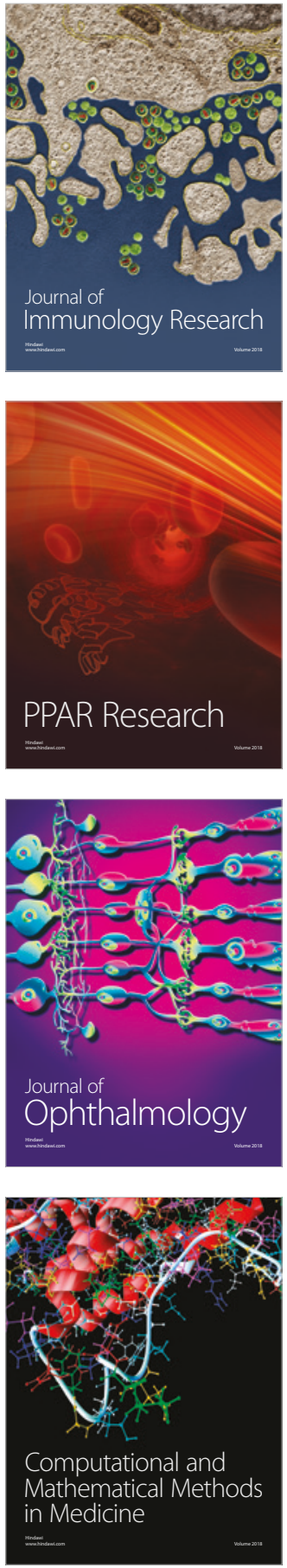

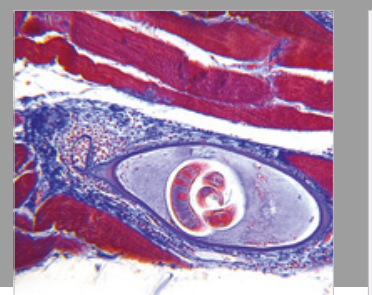

Gastroenterology Research and Practice

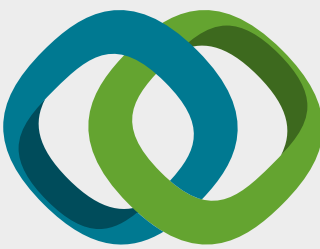

\section{Hindawi}

Submit your manuscripts at

www.hindawi.com
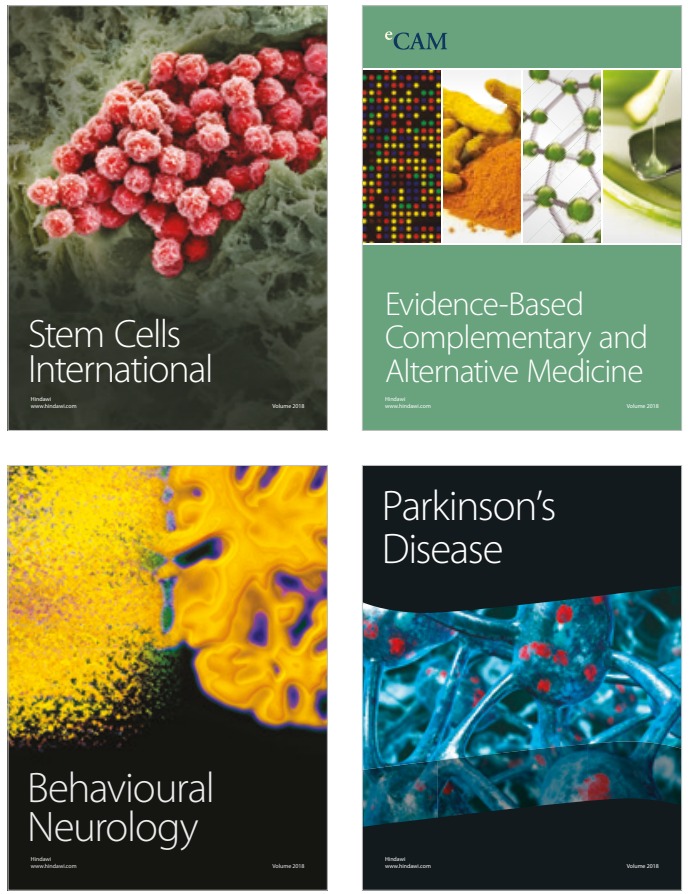

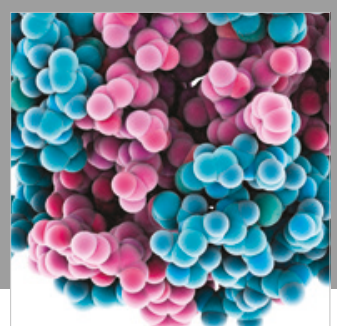

ournal of

Diabetes Research

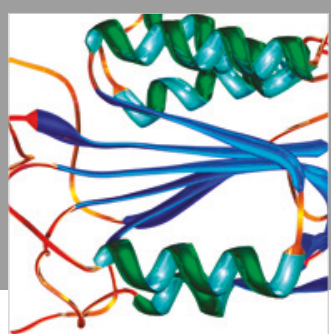

Disease Markers
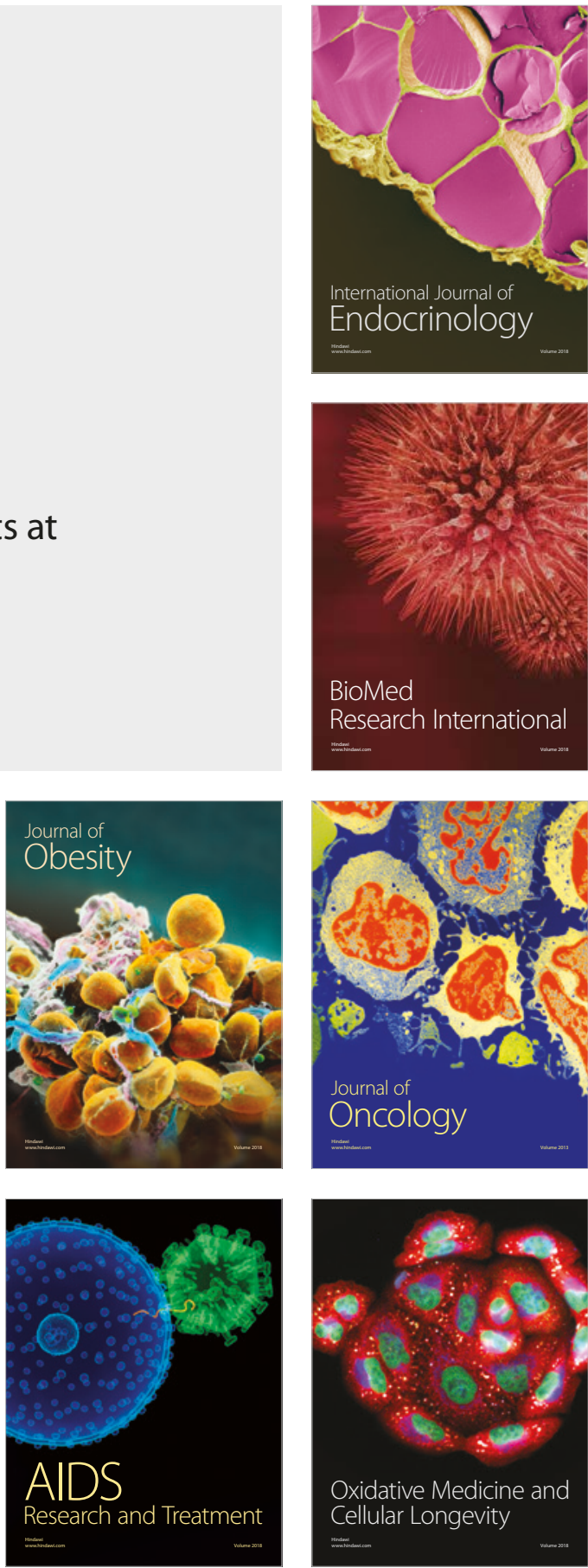